\title{
Distribution of Cytochrome-like Respiration in Streptococci
}

\author{
By T. W. RITCHEY* AND H. W. SEELEY, JR \\ Laboratory of Microbiology, Cornell University, Ithaca, New York 14853 , U.S.A.
}

(Received 7 March 1975; revised 27 August 1975)

\begin{abstract}
SUMMARY
The electron transport systems of I34 strains of streptococci were studied after aerobic growth on glucose in the presence of haematin, by examining the inhibition of electron transport as well as the cellular site of NADH oxidation. Each strain was placed into one of three possible groups: cytochrome-like NADH oxidase; flavin-like NADH oxidase; or no NADH oxidase. Most $(88 \%)$ of the strains of Streptococcus faecalis and its variants liquefaciens and zymogenes and a few strains of $S$. lactis and its variant diacetylactis contained cytochrome-like respiratory systems. Other streptococci including $S$. faecium fell into one of the other groups but did not contain cytochrome-like NADH oxidases.
\end{abstract}

\section{INTRODUCTION}

None of the members of the genus Streptococcus is known to be capable of haem synthesis. When grown in a glucose-yeast extract medium neither catalase nor cytochromes are produced. Whittenbury (1964), Bryan-Jones \& Whittenbury (1969), Sijpsteijn (1970) and Ritchey \& Seeley (I974a) have shown, however, that cytochromes are formed in some streptococci grown aerobically in media containing haem compounds. These organisms are blocked in the steps of haem synthesis but possess the genetic determinants to establish a membrane-bound cytochrome electron transport chain under appropriate conditions.

Ritchey \& Seeley (1974a) demonstrated functional cytochromes in Streptococcus faecalis var. zymogenes by measuring molar growth yields and in vitro $\mathrm{P}: \mathrm{O}$ ratios from membrane extracts. The cytochrome content of cells was also examined spectrophotometrically. Because of their complexities, however, these methods were unsatisfactory for a large-scale survey of a number of species. By investigations of the cellular site of NADH oxidase, and the selective inhibition of this enzyme pathway, the incidence of a cytochrome-like system in streptococci is demonstrated.

\section{METHODS}

Bacteria. The streptococci studied are listed in Table I. The sources of strains are given where known. Strain identification was verified by a large number of physiological and biochemical tests.

Growth and harvesting of bacteria. Bacteria were grown in 21 baffled flasks containing $450 \mathrm{ml}$ medium of initial $\mathrm{pH} 7 \cdot 6$, containing $(\mathrm{g} / \mathrm{l})$ : tryptone (Difco), 10 ; yeast extract (Difco), 5 ; glucose, $4 ; \mathrm{K}_{2} \mathrm{HPO}_{4}$, 3. Haematin $(0.5 \mathrm{~g} / 100 \mathrm{ml}$ of $0.2 \mathrm{M}-\mathrm{KOH})$ was prepared monthly by filter sterilization and added to a final concentration of $20 \mu \mathrm{g} / \mathrm{ml}$. The inoculum was $9 \mathrm{ml}$ from a 10 to $20 \mathrm{~h}$ culture. The flasks were incubated at $30^{\circ} \mathrm{C}$ with shaking until

\footnotetext{
* Present address: Lever Brothers, Co., Research and Development Division, Edgewater, New Jersey 07020 , U.S.A.
} 


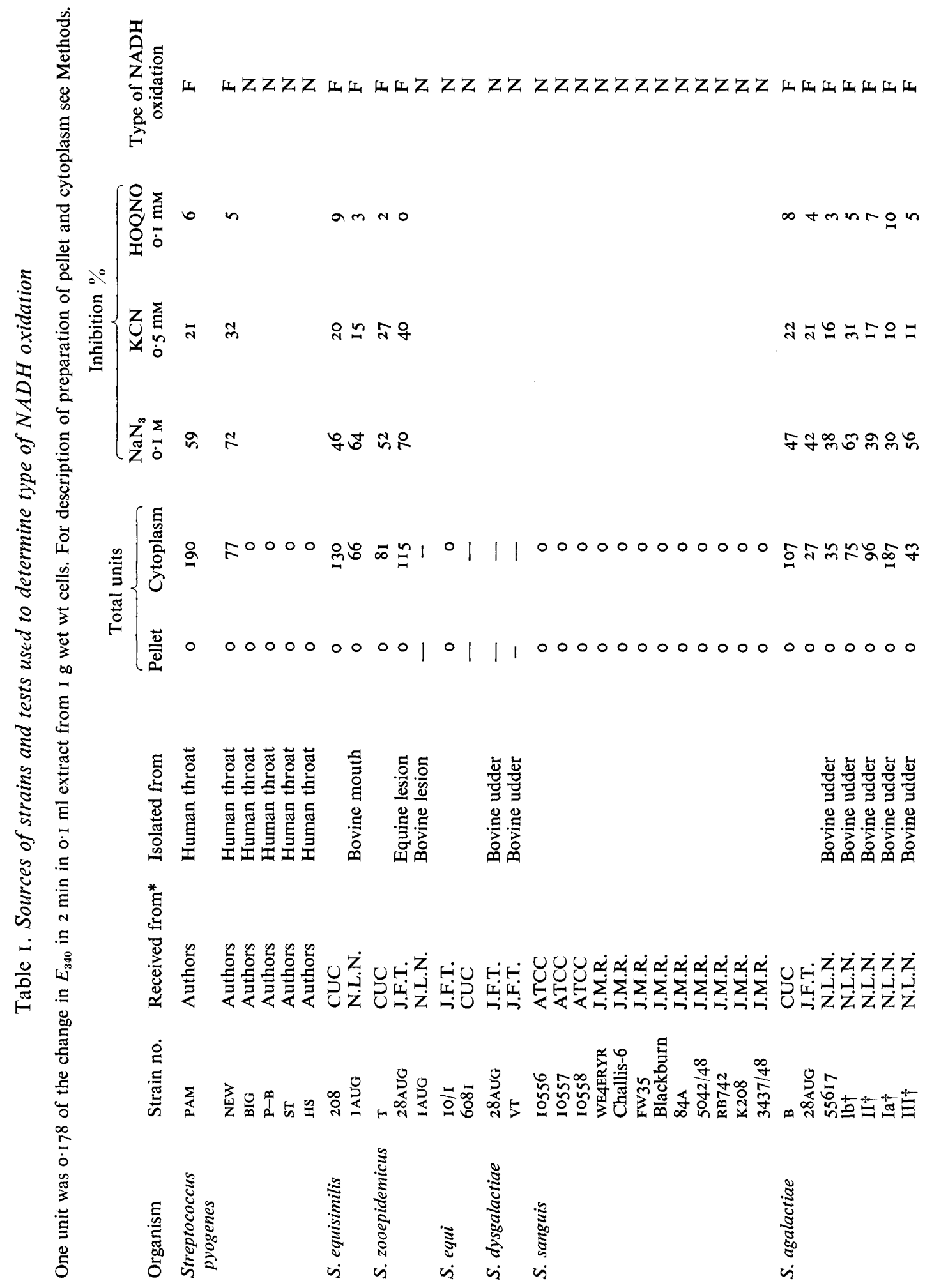




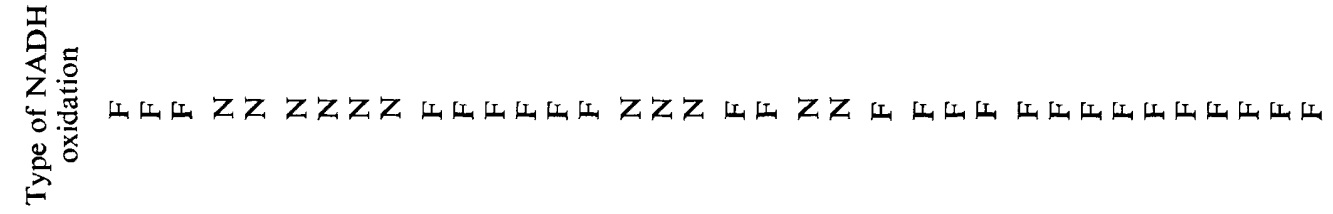

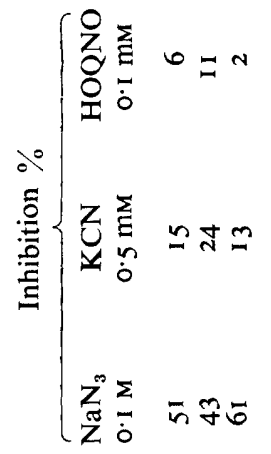
$n \infty m=N$
$\infty n$

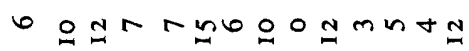

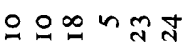
$\infty \cong \pi$ ก

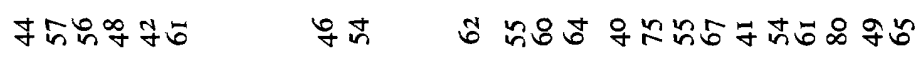

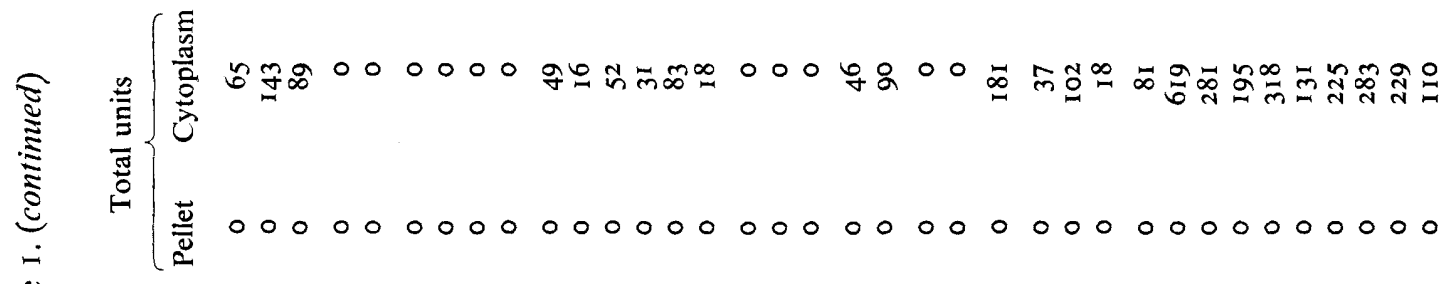

$\stackrel{*}{\Xi}$

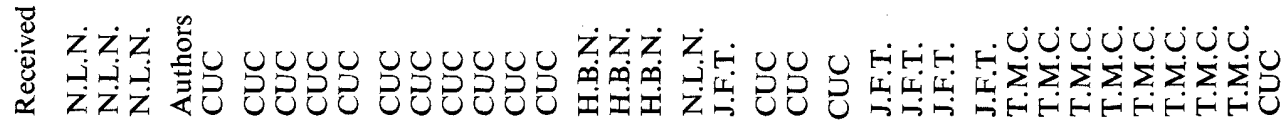

总

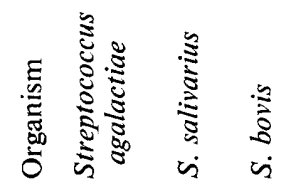

离

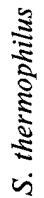

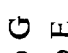

奂 䒓

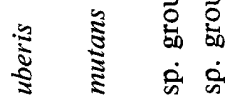

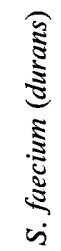



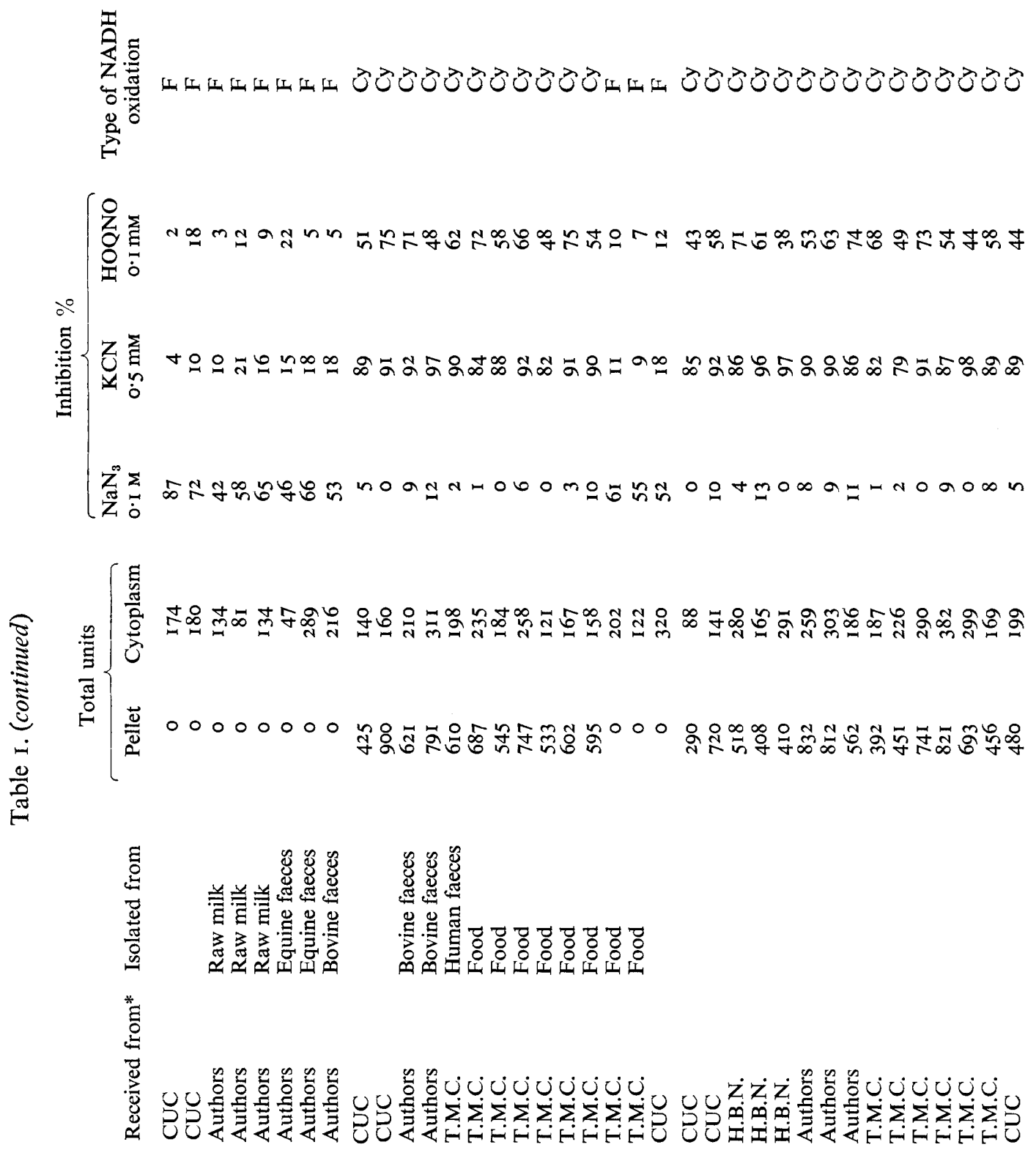

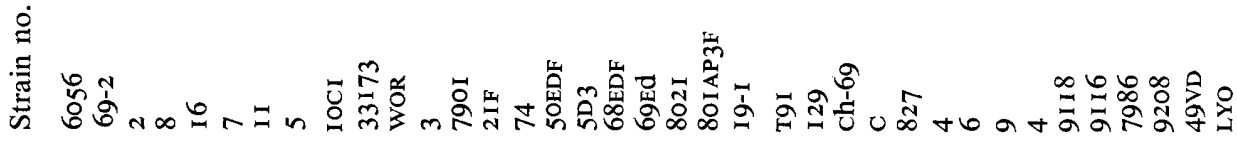

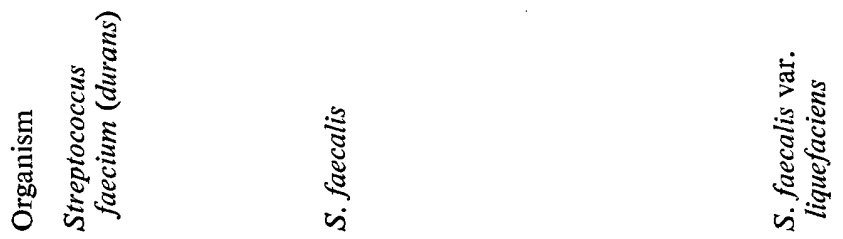




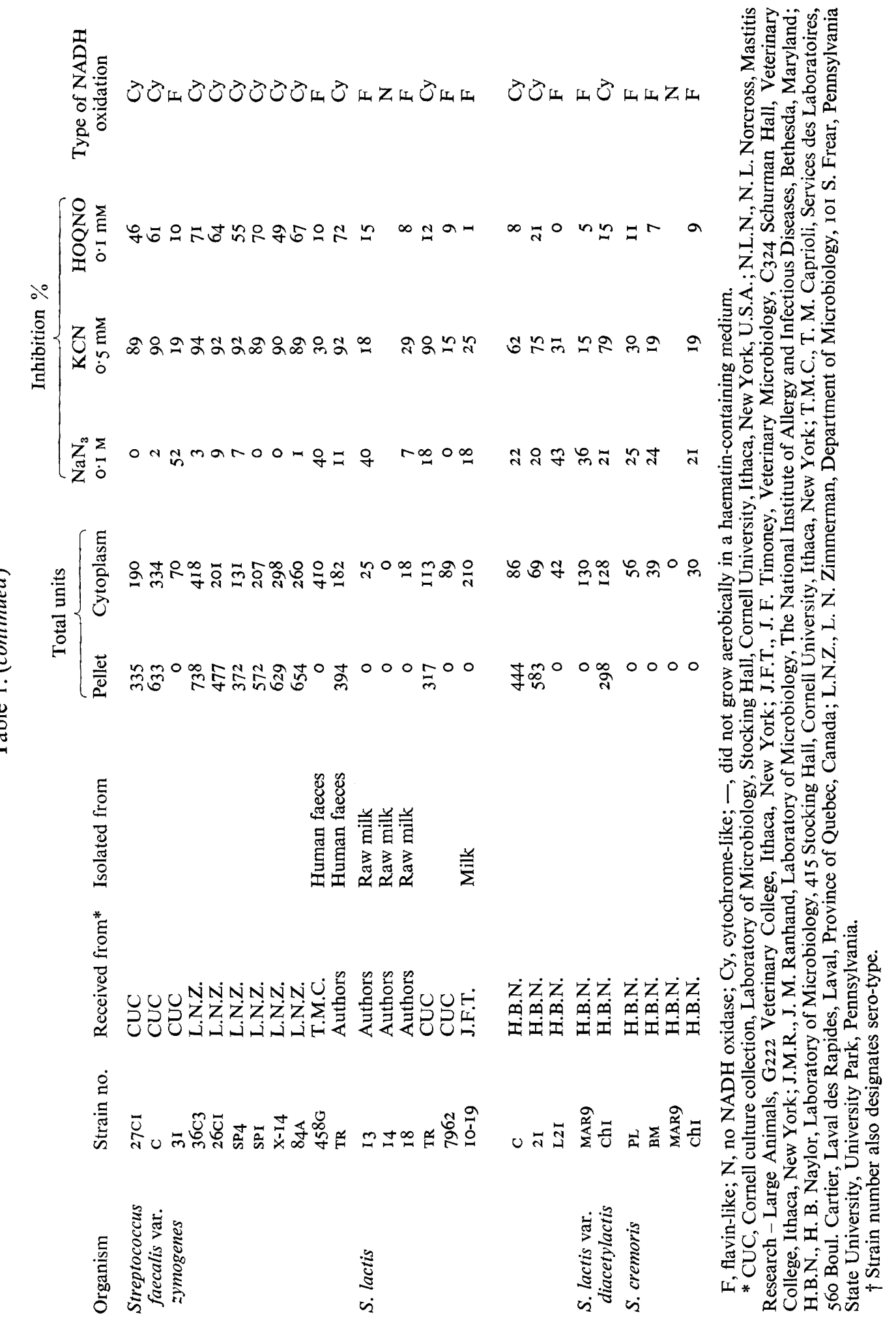


the late-exponential phase of growth was reached. Since generation times varied for different species the time of incubation was altered to give a constant final growth state. Bacteria were harvested by centrifuging at $15000 \mathrm{~g}$ for $\mathrm{I} 5 \mathrm{~min}$ (2 I $500 \mathrm{~g}$ for I $5 \mathrm{~min}$ was used where cell packing was insufficient), then washed twice in $0.015 \mathrm{M}-2-n$-morpholino-ethanesulphonic acid (MES) at $\mathrm{pH} 7 \cdot 3$.

Bacterial extracts. Bacteria (I g wet wt) were resuspended in $25 \mathrm{ml} \mathrm{MES}$ and then broken in an X-Press (BBL, Rockville, Maryland, U.S.A.) at $-25^{\circ} \mathrm{C}$ and $20000 \mathrm{lb}^{-2}$ (I $38 \mathrm{MPa}$ ). The resulting suspension was centrifuged at $40000 \mathrm{~g}$ for $\mathrm{I} 5 \mathrm{~min}$ and the pellet discarded. The supernatant fluid was recentrifuged at $78000 \mathrm{~g}$ for $90 \mathrm{~min}$. The supernatant fluid from this centrifugation contained the cytoplasm. The pellet (resuspended in $5 \mathrm{ml} \mathrm{MES} \mathrm{with} 10 \%$ sucrose) contained the vesicles and small membrane fragments. Certain strains and particular species were harder to break than others, and this introduced an inaccuracy in that a preparation with high breakage liberated more units of NADH oxidase into the cell-free fraction. Between 50 and $80 \%$ of the bacteria from any species were broken by the X-Press. Thus, if a strain was reported to have 400 units of NADH oxidase/g wet wt the maximum error was $\pm \mathrm{I} 20$ units. The variation did not affect the inhibitor data or data on the site of cellular NADH oxidase. Duplicate breakage of a single strain indicated that no more than $\pm 10 \%$ variation would result.

NADH oxidase activity. The assay for NADH oxidase activity was performed as previously described (Ritchey \& Seeley, I $974 a$ ). If the primary assay of $0 \cdot \mathrm{I} \mathrm{ml}$ of the membrane fraction showed very high levels of activity, the $5 \mathrm{ml}$ membrane fraction was diluted. Inhibitors of NADH oxidation $\left[0.20 \mathrm{ml}\right.$ of $\mathrm{I} \cdot 0 \mathrm{M}-\mathrm{NaN}_{3}$ to give a final concentration of $0 . \mathrm{I} \mathrm{M}, 0.05 \mathrm{ml}$ of $0.02 \mathrm{M}-\mathrm{KCN}$ to give a concentration of $0.5 \mathrm{mM}$, or $5.0 \mu \mathrm{l}$ of $2-n$-heptyl-4hydroxyquinoline- $n$-oxide (HOQNO) to give a final concentration of $0 \cdot 1 \mathrm{~mm}$ ] were added in three subsequent assays.

The $25 \mathrm{ml}$ fraction containing the cytoplasm was then assayed for enzymic activity. Normally $0.2 \mathrm{ml}$ of the $25 \mathrm{ml}$ fraction contained the optimum concentration of activity for these assays. Since inhibitors were used to confirm the predominant type of NADH oxidase, their effect on the cytoplasmic NADH oxidase was not examined if the membrane fraction contained a cytochrome-linked NADH oxidase. The term 'NADH oxidase' is used in a very broad sense and refers to the primary pathway from NADH to oxygen, which is either flavin-linked or cytochrome-linked. Descriptive terms used here to designate these pathways are 'flavin-like' or 'cytochrome-like'. These terms are used to recognize the limitations of classifying $\mathrm{NADH}$ oxidases in specific categories where complete individual electron carrier systems were not isolated and characterized. If the cytochrome-like pathway was not present in the $5 \mathrm{ml}$ membrane fraction, inhibitors were used to confirm the presence of any flavin-like NADH oxidase.

\section{RESULTS}

The NADH oxidase activity was measured in the membrane fraction and cytoplasm of the 134 strains of streptococci (Table 1). A few strains did not grow aerobically under these conditions, although they grew anaerobically in the same medium. In some instances neither the particulate nor the soluble fraction of aerobically haematin-grown cells contained any NADH oxidase activity. These strains were regrown and harvested in the midexponential phase of growth. An organism was classified as not containing NADH oxidase (group 3) if activity could not be found under any of these conditions.

An organism was classified as flavin-like (group 2) if fewer than 10 units of enzyme activity were present in the $5 \mathrm{ml}$ of the particulate fraction (this represented the lower limit 
of assay sensitivity) and there was detectable soluble NADH oxidase. The criteria used to classify either pathway were arbitrary since a precedent for such a scheme has not yet been established. The limits for assigning either units of activity or percentage inhibition were determined by careful examination of the data. The presence of a flavin-like system was confirmed by measuring inhibition of the rate of $\mathrm{NADH}$ oxidation. With $0 \cdot \mathrm{I} M-\mathrm{NaN}_{3}$, inhibition was greater than $35 \%$, with $0.5 \mathrm{~mm}-\mathrm{KCN}$ it was less than $35 \%$, and with $0.1 \mathrm{~mm}-$ HOQNO less than $25 \%$. The species which were difficult to fit into this classification were Streptococcus lactis, S. lactis var. diacetylactis and S. cremoris. These species (the lactic streptococci) may have a different type of flavin-linked NADH oxidase, in that their soluble NADH oxidase was relatively insensitive to $\mathrm{NaN}_{3}$.

An organism was labelled 'cytochrome-like' (group I) if there were more than 250 units of enzymic activity present in the $5 \mathrm{ml}$ of the particulate fraction. The presence of a cytochrome system was confirmed by the degree of inhibition of the rate of NADH oxidation. With $0.1 \mathrm{M}-\mathrm{NaN}_{3}$, inhibition was less than $20 \%$, with $0.5 \mathrm{mM} \mathrm{KCN}$ it was greater than $75 \%$, and with $0 . \mathrm{I} \mathrm{mM} \mathrm{HOQNO}$ greater than $35 \%$. Again the lactic streptococci presented the exceptions: those with a cytochrome-like NADH oxidase system were not very sensitive to HOQNO.

Streptococci were divided into six general groups, depending upon the type of NADH oxidase found. (i) The species which failed to reveal any type of NADH oxidase: $S$. equi, $S$. dysgalactiae, S. sanguis, S. salivarius, S. bovis, S. thermophilus and S. mutans. (ii) The species containing strains, some of which were devoid of NADH oxidase and others with a flavin-type NADH oxidase: $S$. pyogenes, $S$. zooepidemicus and $S$. cremoris. (iii) The species with strains which contained the flavin-type NADH oxidase alone: $S$. equisimilis, S. agalactiae, S. equinus, S. uberis, Streptococcus group G, Streptococcus group E, and $S$. faecium (including strains formerly known as $S$. durans). (iv) $S$. faecalis var. liquefaciens, the only species which contained strains with a cytochrome-like NADH oxidase alone. (v) Strains of S. faecalis, S. faecalis var. zymogenes and S. lactis var. diacetylactis which produced either the cytochrome-like or the flavin-like NADH oxidase systems. The strains of $S$. faecalis and $S$. faecalis var. zymogenes which produced the flavin-like system were old laboratory cultures, by which is meant a strain which had been isolated more than one year prior to this study and which had usually been carried in laboratory media for many sub-transfers. The term old laboratory culture is used to call attention to the fact that mutations might have occurred during the time of sub-culturing, thus affecting components necessary for the cytochrome pathway. (vi) S. lactis the only species which contained strains which fell into the main groups $i$, ii, and iv.

\section{DISCUSSION}

The ability of certain streptococci to metabolize haem compounds has remained obscure until recently because good growth occurred in many media without haematin and where cytochromes did appear they were inducible. In the present study all strains which produced cytochromes in haematin-containing medium also produced a flavin system when grown in a medium devoid of haematin, and were thus haematin-dependent for cytochrome formation but not for respiration.

The object of this investigation was to identify species of the genus Streptococcus able to form cytochromes when grown under certain defined conditions. Using cell-free extracts of newly isolated strains, the distinction between the cytochrome and flavin systems was easily made. Cytochrome-like enzymic activity, when it occurred, was present in the washed membranes at levels of at least 290 units as seen in $S$. faecalis var. liquefaciens strain T9I 
(Table I). This activity was significantly more sensitive to $\mathrm{KCN}$ and HOQNO but less sensitive to $\mathrm{NaN}_{3}$ than was the activity of the flavin system.

Under our cultural conditions, several strains produced less than 50 units of flavinlinked NADH oxidase/g wet wt bacteria. These strains were only weakly oxidative, and would have been classified as lacking NADH oxidase if particular attention had not been given to cultivation of batches. Several species were found to grow aerobically only if haematin were present. This effect was not due to the induction of cytochromes, however, since NADH oxidase was not produced. Ritchey \& Seeley (I974b) have reported that I3 strains of $S$. sanguis were protected from hydrogen peroxide in the presence of haematin under aerobic conditions. These strains gave increased molar growth yields but did not produce cytochromes.

Of the enterococci (all serological group D) only $S$. faecalis and its two varieties produced the cytochrome-like system. All 18 strains of $S$. faecium failed to form cytochromes. Of the 40 strains of the $S$. faecalis group only five did not form cytochromes. These five strains were old laboratory cultures which may have been genetically altered. A block at any site of the cytochrome pathway would result in the establishment of a mutant strain. The number of loci needed to code for an entire cytochrome system must be large, thus increasing the chances for the occurrence and persistence of mutants incapable of cytochrome induction. This possibility is increased since the maintenance of a culture in the laboratory is likely to have been in haematin-free media. It should be noted, however, that several old laboratory cultures produced the cytochrome system.

Although it was not our purpose to examine the types of flavin-like electron transport systems in the streptococci, sufficient evidence was gathered to conclude that the lactic streptococci may have a flavin system differing from those of other streptococci since it was less sensitive to $\mathrm{NaN}_{3}$. Of I5 strains of the lactic streptococci (all serological group $\mathrm{N}$ ), four formed cytochromes. The other strains contained either a flavin NADH oxidase or no NADH oxidase.

London \& Kline (1973), upon examining the aldolases of several species of the genera Streptococcus, Lactobacillus and Pediococcus, found that the electrophoretic mobility rates of the fructose diphosphate aldolases of $S$. faecalis strain MR and $S$. cremoris strain ATCCI9259 were somewhat similar but they differed equally from $S$. durans ( $S$. faecium). This would seem to relate to our finding that $S$. faecalis and the lactic streptococci form cytochromes but $S$. faecium does not. London \& Kline (1973) presented a phylogenetic map which placed the enterococci and the group $\mathrm{N}$ streptococci near the genera Lactobacillus and Pediococcus, in a linkage closer than to any other member of the genus Streptococcus. These findings are in general agreement with those of Whittenbury (1965) which demonstrated a close linkage between pediococcus and enterococcus. Species of the genus Pediococcus, however, appeared to be physiologically and biochemically more like $S$. faecium than $S$. faecalis. Furthermore, $S$. faecalis but not $S$. faecium produces cytochromes when grown in a haematin-containing medium. Additional work may be needed to reclassify $S$. faecalis at the genus level. The ability of some strains of $S$. lactis to form cytochromes suggests ancestral linkages not too remote from $S$. faecalis. Although Bergey's Manual of Determinative Bacteriology (1974) states that members of the genus Streptococcus do not contain haem compounds, this view must be modified to recognize that $S$. faecalis and $S$. lactis may contain cytochromes in appropriate conditions. The extent to which cytochrome formation occurs under natural conditions, e.g. during infections where suitable haem precursors for cytochrome formation are available, remains to be determined.

Cytochrome induction in streptococci has been shown to occur exclusively under aerobic 
conditions and is dependent upon the environment for exogenous haem. Van der. WielKorstanje \& de Vries (1973), using another faecal inhabitant, Bifidobacterium longum, found that lysed red blood cells induced cytochromes $b$ and $a$ aerobically or cytochromes $b$ and $d$ anaerobically during nitrate reduction. These findings parallel the data on cytochrome induction in streptococci to the extent that in both cases a haem compound was necessary for aerobic cytochrome formation. If an anaerobic cytochrome system exists in S. faecalis, it has not yet been demonstrated; however, the conditions of the intestine have not been simulated, since an alternate electron acceptor such as nitrate has never been provided. Langston \& Williams (1962) reported that some group D streptococci were capable of nitrate reduction.

Another faecal inhabitant, Escherichia coli, contains aerobic cytochromes as well as anaerobic cytochromes for nitrate reduction. The aerobic cytochromes are induced by exposure to highly oxidative conditions and produce additional energy for growth. Cytochromes may confer a similar advantage upon $S$. faecalis. Streptococcus faecalis var. zymogenes produces $4 \mathrm{~mol} \mathrm{ATP} / \mathrm{mol}$ glucose aerobically with the flavin electron transport system (Ritchey \& Seeley, I974a). With the cytochrome system, 5 mol ATP were produced. Since the flavin system may be non-functional] in an anaerobic environment, only 2 mol ATP would be formed from fermentation. If these organisms had an anaerobic cytochrome system, however, the additional energy yield, possibly 5 instead of 2 mol ATP, would be of enormous advantage.

A significant finding (Smalley, Jahrling \& VanDemark, I968) was that $S$. faecalis strain IOC I was capable of oxidative phosphorylation without haematin. We found that cytochromes were induced when this organism was grown with haematin but that the energy yield was only slightly increased. The aerobic molar growth yield of 53.5 with the flavin system increased only to $56 \cdot 2$ with the cytochrome system. It appears that the cytochrome system which developed in the presence of haematin was favoured over the flavin system possibly because the former has a higher capacity for binding most terminal electron acceptors (Niederpruem \& Hackett, 1958).

\section{REFERENCES}

Bergey's Manual of Determinative Bacteriology, 8th edn (1974). Edited by R. E. Buchanan and D. E. Gibbons. Baltimore: The Williams and Wilkins Co.

Bryan-Jones, D. G. \& WhitTenbury, R. (1969). Haematin-dependent oxidative phosphorylation in Streptococcus faecalis. Journal of General Microbiology 58, 247-260.

Langston, C. W. \& Williams, P. P. (1962). Reduction of nitrate by streptococci. Journal of Bacteriology 84,603 .

London, J. \& KLINE, K. (1973). Aldolase of lactic acid bacteria, a case history in the use of an enzyme as an evolutionary marker. Bacteriological Reviews $37,453-478$.

Niederpruem, D. J. \& Hackett, D. P. (1958). The oxygen affinity of a flavin oxidase involved in the respiration of Streptococcus faecalis. Plant Physiology 33, I I 3-1 I 5.

Ritchey, T. W. \& SEeley, H. W. (I 974a). Cytochromes in Streptococcus faecalis var. zymogenes grown in a haematin-containing medium. Journal of General Microbiology 85, 220-228.

Ritchey, T. W. \& SeEley, H. W. (1974b). Growth of Streptococcus sanguis in a defined medium. Abstracts of the Annual Meeting of the American Society for Microbiology, 42.

SIJPSTEIJN, A. K. (1970). Induction of cytochrome formation and stimulation of oxidative dissimilation by hemin in Streptococcus lactis and Leuconostoc mesenteroides. Antonie van Leeuwenhoek 36, 335-348.

Smalley, A. J., Jahrling, P. \& VanDemark, P. J. (I968). Molar growth yields as evidence for oxidative phosphorylation in Streptococcus faecalis strain IOCI. Journal of Bacteriology 96, I595-1600.

VAN DER Wiel-Korstanje, J. A. A. \& DE VRIEs, W. (I973). Cytochrome synthesis by Bifidobacterium during growth in media supplemented with blood. Journal of General Microbiology 75, 417-4I9.

Whittenbury, R. (I964). Hydrogen peroxide formation and catalase activity in the lactic acid bacteria. Journal of General Microbiology 35, 13-26.

Whittenbury, R. (I965). A study of some pediococci and their relationship to Aerococcus viridans and the enterococci. Journal of General Microbiology 40, 97-106. 